\title{
Influence of Surfactants on Lipase Fat Digestion in a Model Gastro-intestinal System
}

\author{
Pedro M. Reis - Thomas W. Raab - Jean Y. Chuat • \\ Martin E. Leser • Reinhard Miller • \\ Heribert J. Watzke $\cdot$ Krister Holmberg
}

Received: 15 January 2008 / Accepted: 21 May 2008 /Published online: 26 June 2008

(C) Springer Science + Business Media, LLC 2008

\begin{abstract}
In the present study, we use a model gastro-intestinal system to study the influence of different food-grade surfaceactive molecules (Sn-2 monopalmitin, $\beta$-lactoglobulin, or lysophosphatodylcholine) on lipase activity. The interfacial activity of lipase and surfactants are assessed with the pendant drop technique, a commonly used tensiometry instrument. A mathematical model is adopted which enables quantitative determination of the composition of the wateroil interface as a function of bulk surfactant concentration in the water-oil mixtures. Our results show a decrease in gastric lipolysis when interfacially active molecules are incorporated into a food matrix. However, only the Sn-2 monopalmitin caused a systematic decrease in triglyceride hydrolysis throughout the gastro-intestinal tract. This effect is most likely due to exclusion of both lipase and triglyceride from the water-oil interface together with a probable saturation of the solubilization capacity of bile with monoglycerides. Addition of $\beta$-lactoglobulin or lysophopholipids increased the hydrolysis of fat after the gastric phase. These results can be attributed to an increasing interfacial area with lipase and substrate present at the interface. Otherwise, $\beta$-lactoglobulin,
\end{abstract}

P. M. Reis · T. W. Raab J. Y. Chuat - M. E. Leser $\cdot$ H. J. Watzke Nestlé Research Center,

CH-1000 Lausanne 26, Switzerland

P. M. Reis $\cdot$ K. Holmberg $(\bowtie)$

Department of Chemical and Biological Engineering,

Applied Surface Chemistry, Chalmers University of Technology,

SE-412 96 Gothenburg, Sweden

e-mail: kh@chalmers.se

R. Miller

Max-Planck-Institut für Kolloid-und Grenzflächenforschung,

Max-Planck-Campus,

D-14476 Golm, Germany or lysophopholipids reduced fat hydrolysis in the stomach. From the mathematical modeling of the interface composition, we can conclude that Sn-2 monopalmitin can desorb lipase from the interface, which, together with exclusion of substrate from the interface, explains the gradually decreased triglyceride hydrolysis that occurs during the digestion. Our results provide a biophysics approach on lipolysis that can bring new insights into the problem of fat uptake.

Keywords Lipase · Fat · Hydrolysis · Emulsion · Digestion · Surfactant $\cdot$ Model gastro-intestinal system .

TNO intestinal model

\section{Introduction}

Dietary fats may be consumed by humans in a variety of different forms, e.g., as bulk fats, fats entrapped in solid matrices, water-in-oil emulsions, or oil-in-water emulsions ${ }^{1}$. However, most fats are converted into oil-in-water emulsions in the mouth, stomach, and/or small intestine due to input of mechanical energy and the presence of surfaceactive molecules originating from food or secreted by the body, such as bile salts, phospholipids, and proteins ${ }^{2,3}$. Therefore, the substrates for lipases are usually fat droplets embedded in an aqueous medium, with the surfaces of the droplets being coated by a complex layer of interfacially active molecules of different origin ${ }^{4}$.

In humans, digestion of dietary triacylglycerols (triglycerides) starts in the stomach where they are typically hydrolyzed to a degree of 10 to $30 \%$ by an acid-stable gastric lipase ${ }^{5}$. This relatively limited extent of lipolysis under physiological conditions suggests a feedback mechanism that inhibits further lipolysis ${ }^{6,7}$. It is known that gastric lipolysis is essential for emulsification as a way of 
increasing the substrate availability for the pancreatic lipase in the duodenum ${ }^{8}$.

An important aspect of lipolysis is the special character of the involved interfacial process, i.e., adsorption of the lipase at the interface followed by catalysis ${ }^{9}$. The extent of lipase binding is related to the physicochemical as well as the compositional structure of the interface, for which the expression "quality of the interface" has been coined ${ }^{10}$. The meaning of "quality" is vague but the concept contains contributions from electrostatic and van der Waals interactions, orientation of the substrate and hydration forces. One would therefore expect that the nature of the interfacial layer surrounding the lipid droplets would play an important role in determining the rate and extent of lipid hydrolysis. Hence, in order to understand the nature of lipolysis, the chemical composition and the physical structure of the substrate-containing interface must be known $^{11}$.

Lipase-surfactant interactions have been extensively studied in the literature ${ }^{12-17}$; however, the inhibition of lipase activity has not been directly related to the interfacial activity of the amphiphiles ${ }^{18}$. It is known that bile and colipase can reverse the inhibitory effect of surfactants on pancreatic lipase. However, this activation effect is limited to a narrow concentration range of the amphiphile ${ }^{18}$.

During digestion, lipases are exposed to a wide variety of interfacially active molecules which either occur naturally in the gastro-intestinal tract, are added into the food, or are generated upon hydrolysis.

In the present work, we evaluate the effect of three interfacially active molecules; Sn-2 monopalmitin (Sn-2 MC16), lysophosphatidylcholine (LysoPC), and $\beta$-lactoglobulin (BLG), on tricaprylin (TC8) lipolysis. The selected amphiphiles may either be present in the digested food or generated during the digestion. A model gastro-intestinal system (TNO Intestinal Model 1, TIM1 from TNO), which simulates the physiological conditions of the human stomach and small intestine, was used in order to monitor the effect of the added interfacially active molecules on the digestion of triglycerides. The system has already been used before to study the influence of partially hydrolyzed guar gum on fat hydrolysis ${ }^{19}$ and fibers on fat absorption ${ }^{20}$, observing good correlation with in vivo results.

The interfacial activity of the tested amphiphiles is assessed in a model water-in-oil system, which allows to assess the interfacial composition, i.e., the effect of the different amphiphiles on the adsorption properties of both lipase and triglyceride at the oil-water interface. For the characterization of the interfacial composition, we have used the mathematical models developed by Fainerman et $^{2} .^{21-23}$. Previous models ${ }^{24}$ do not take into account the ability of protein molecules to occupy variable (depending on the surface coverage) area in the surface layer, and also do not involve the nonideality of enthalpy in the formation of mixed layers. New evidence show ${ }^{24}$ that there is a significant contribution from the nonideality of entropy in the process of competitive adsorption. In particular, the ability of small molecules to displace macromolecules from the adsorption layers is due to this effect.

In the present work, expressions were used for the entropy of mixing of molecules occupying different areas in the surface layer, for the surface tension of mixed solutions, and for the adsorption of both types of molecules.

\section{Materials and Methods}

\section{Gastro-intestinal Model}

A dynamic in vitro gastro-intestinal model (TIM) was used to evaluate the influence of surface-active molecules on lipolysis of triglycerides by gastric and pancreatic lipases. The TIM model was developed by TNO (Zeist, The Netherlands) and previously described and patented by Minekus et al. ${ }^{25,26}$. This system mimics the major events occurring in the lumen of the gut, thus allowing studies of the effects preceding absorption through the gut wall. Validation studies with this system on the availability of fat soluble vitamins from processed vegetables show a good relation with in vivo data ${ }^{27,28}$. Furthermore, the system has been used to study the effect of partially hydrolyzed guar gum, on the bioaccessibility of fat and cholesterol ${ }^{19}$. The model consists of four serial compartments simulating the stomach, duodenum, jejunum, and ileum (Figure 1). The jejunum and ileum compartments are each connected to filtration units (Spectrumlabs Minikros ${ }^{\circledR}$, M20S-300-01P). After the ileal valve, the efflux is collected.

\section{Experimental Conditions}

A model test meal is used in our experiments, which consists of a tricaprylin/water emulsion with a selected surfactant. Starch is incorporated in all the different samples in order to suppress phase separation before starting the digestion process. The direct study of complex matrixes that resemble real food systems would lead to inconclusive data due to the presence of other substances that can affect fat digestion (e.g., proteins, salts). A well-defined system with a single variable is therefore suited for a good understanding of the influence of the surfactant type on fat digestibility.

The meals were prepared as follows: a buffer solution of $200 \mathrm{ml}$ sodium phosphate $(20 \mathrm{mM}$; pH 5.5) was heated to $100^{\circ} \mathrm{C}$, a $25-\mathrm{ml}$ starch solution containing $10 \mathrm{~g}$ starch from potato (Sigma), was added and the mixture was cooled down to $37^{\circ} \mathrm{C}$. Eight grams of TC8 ( $\geq 99 \%$, Sigma) was added together with either $1.00 \mathrm{~g}$ of $\mathrm{Sn}-2 \mathrm{MC} 16(\geq 99 \%$, 
Fig. 1 Schematic diagram of the in vitro gastro-intestinal model, TIM: $(A)$ gastric compartment; $(B)$ duodenal compartment; $(C)$ jejunal compartment; $(D)$ ileal compartment; $(E)$ glass jacket; $(F)$ flexible wall; $(G)$ rotary pump; $(H)$ pyloric valve; $(I) \mathrm{pH}$ electrodes; $(J)$ secretion pump; $(K)$ pre-filter; $(L)$ hollow fiber membrane; $(M)$ filtrate from jejunum; $(N)$ ileal delivery valve

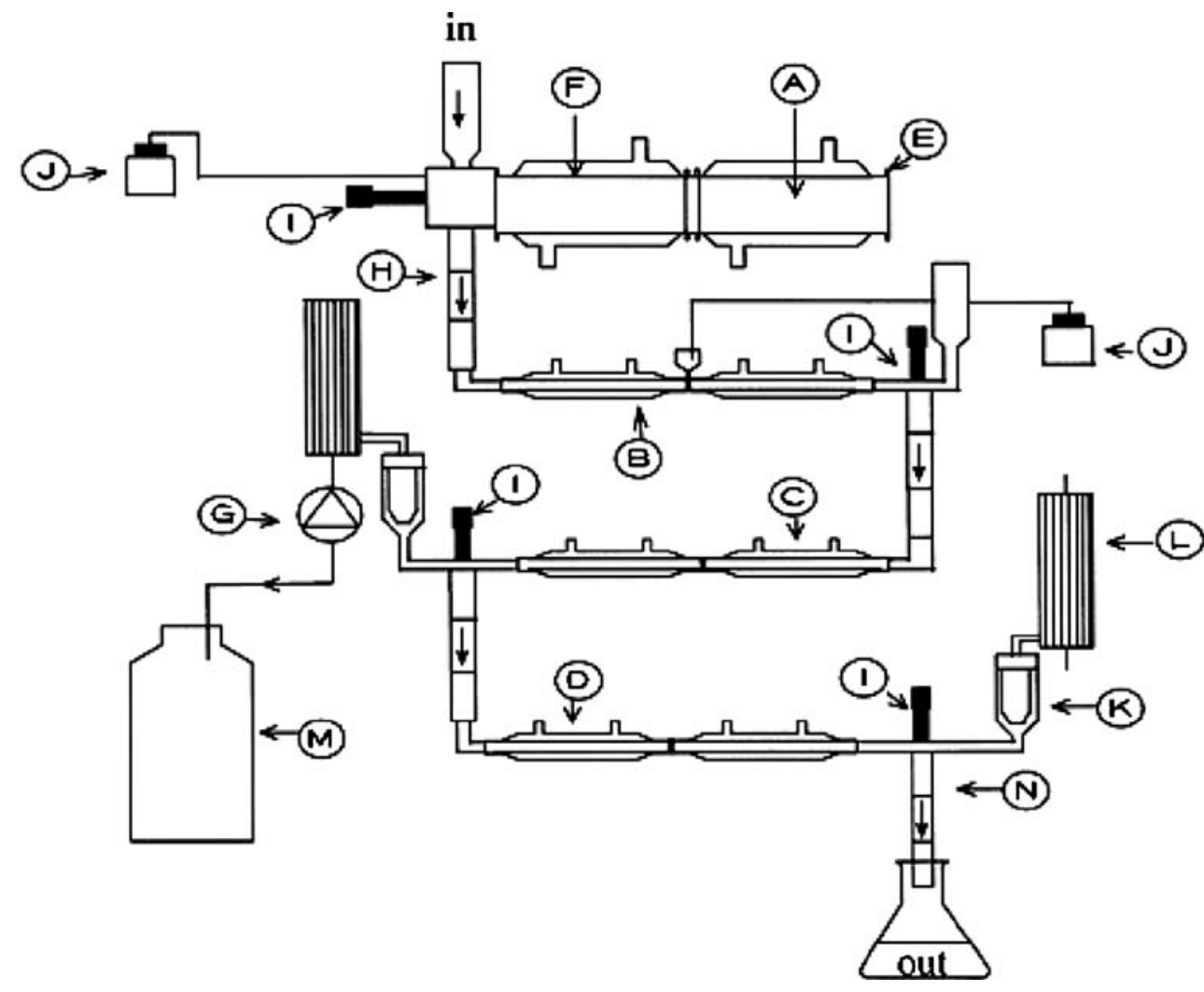

set-points of $6.5,6.8$, and 7.2. The duodenal compartment was filled with a solution consisting of $15 \mathrm{~g}$ SIES, $15 \mathrm{~g}$ of a $7 \%$ pancreatin solution, $30 \mathrm{~g}$ of fresh porcine bile, and $2 \mathrm{mg}$ of trypsin (Sigma, T4665-5G) prior to the experiment to simulate initial intestinal contents. The jejunal compartment was filled with a solution consisting of $30 \mathrm{~g}$ SIES, $30 \mathrm{~g}$ of a $7 \%$ pancreatin solution, and $60 \mathrm{~g}$ of fresh porcine bile. The ileal compartment was filled with SIES solution.

During the experiments, samples were taken directly from the lumen of the different compartments and filtrates, as well as the efflux, were collected.

\section{Extraction of Free Fatty Acids}

Chloroform $(900 \mu \mathrm{l})$ and $0.1 \mathrm{M} \mathrm{HCl}(100 \mu \mathrm{l})$ were added to an aliquot of sample $(100 \mu \mathrm{l})$. The mixture was vortexed for $1 \mathrm{~min}$ followed by centrifugation at $15,000 \mathrm{rpm}$ for $10 \mathrm{~min}$. After extraction and complete phase separation, $500 \mu \mathrm{l}$ of the apolar phase were transferred into a new vial and directly analyzed by gas chromatography (GC) coupled with a flame ionization detection system (FID).

\section{GC-FID}

Free fatty acids were analyzed using an Agilent 6890 gas chromatograph equipped with a flame ionization detection system. Separation of the surface-active molecules was carried out in a non-polar fused silica column $(30 \mathrm{~m}$ long 
with an inner diameter of $0.25 \mathrm{~mm}$ ) coated with methylphenylpolysiloxane (DB-5 HT from J\&W). Sample injection $(1 \mu \mathrm{l})$ was performed directly on the column with the injector tracking oven temperature. The initial oven temperature was set to $90^{\circ} \mathrm{C}$ for $2 \mathrm{~min}$, followed by an increase of $10^{\circ} \mathrm{C} / \mathrm{min}$ during $8 \mathrm{~min}$. The detector temperature was kept constant at $360^{\circ} \mathrm{C}$. Recording of chromatograms was performed with an HP-Chemstation for GC Systems (Software Chemstation, version A10.01). Chromatographic peaks were identified by comparing the retention times with those of known standards.

\section{Tensiometry (Pendant Drop Technique)}

Interfacial tension was recorded on a Tracker tensiometer from Teclis/IT Concept. A syringe of $250 \mu \mathrm{l}$ from Hamilton (1001 TLLWS) was used with a needle of $1.83 \mathrm{~mm}$ diameter. If not otherwise mentioned, the aqueous phase was held in the syringe, while the apolar phase was kept in a HEL-101-OS-20T cell from Hellma. The principle of the drop profile analysis is based on determination of the coordinates of a liquid drop from a video image and comparison of these coordinates with theoretical profiles calculated from the Gauss-Laplace equation. A detailed description of this methodology was published recently ${ }^{30}$. All measurements were performed using the area mode control of an aqueous drop of $2.97 \mathrm{~mm}^{3}$ volume.

The enzymes adopted to perform tensiometry assays were lipase from Rhizomucor miehei 4,210 units/mg solid (Sigma, St. Louis, MO), since it is free of bile and other contaminating proteins and a crude preparation of lipase from porcine pancreas (100-400 units/mg protein).

\section{Interfacial Shear Rheology (ISR)}

The interfacial shear viscosity was measured with the ISR1 (SINTERFACE Technologies, Berlin) shear rheometer. The principle of these measurements is based on a biconical disk (measurement body) mounted at a torsion wire ${ }^{31}$. The torsion wire is fixed to a deflection drive (a stepper motor). By an instantaneous movement of the torsion head, a torque is applied to the measurement body placed in the planar fluid interface of water/oil in a measuring vessel. A shear field is built up between the inner wall of the measuring vessel and the outer part of the measurement body (sharp edge of biconical disk). The angular displacement of the biconical disk is recorded by the instrument. After deflection, the pendulum performs damped harmonic oscillations with a damping factor $\alpha$ and a radian frequency $\beta$. The angular position of the measurement body is registered via a mini laser and a position-sensitive photosensor with an accuracy of $0.01^{\circ}$. The registered oscillation curve is then fitted by a model in order to determine the damping factor $\alpha$ and the radian frequency $\beta$ of the torsion oscillation. From the difference between the measured values and those obtained for oscillation at a pure water/ decane interface, the rheological parameters are calculated using the following formulae:

$\eta_{\mathrm{S}}=2 H_{\mathrm{s}} I_{\mathrm{r}}\left(\alpha-\alpha_{0}\right)$

$G_{\mathrm{S}}=H_{\mathrm{S}} I_{\mathrm{r}}\left(\alpha^{2}-\alpha_{0}^{2}+\beta^{2}-\beta_{0}^{2}\right)$

where

$\eta_{\mathrm{S}} \quad$ interfacial shear coefficient of viscosity

$G_{\mathrm{S}}$ interfacial shear modulus of rigidity

$H_{\mathrm{s}}$ apparatus constant, depending on the shear field geometry

$I_{\mathrm{r}}$ moment of inertia of the measurement system

$\alpha$ damping factor

$\beta \quad$ frequency of the torsion oscillation

Every $10 \mathrm{~min}$, a measurement of interfacial shear rheology was performed with a duration of about $25 \mathrm{~s}$ per single oscillation measurement. The measurements were performed in a temperature-controlled room at $20^{\circ} \mathrm{C}$ using a deflection angle of $1.5^{\circ}$. A calibration measurement at the pure water/decane interface was used to estimate the values for $\alpha_{0}$ and $\beta_{0}$. These measurements were carried out for more than $2 \mathrm{~h}$ in order to monitor the eventual adsorption or desorption of interfacially active impurities to or from the liquid/liquid interface. The non-aqueous phase must be fixed at the sharp edge of the biconical disk in order to prevent any wetting of the upper part of disk. After the positioning of the biconical disk, the aqueous phase and the disk are covered with the second fluid to a constant level. Exactly the same filling levels must be used for all measurements with interfacially active compounds as for the reference measurement. The measurements were performed as a function of adsorption time with $t=0$ set when the filling procedure was finished.

\section{Mathematical Modeling}

The interfacial area and adsorption constants were determined as follows:

Low-molecular-weight Surfactants

\section{Langmuir Model}

The equation of state (3) and adsorption isotherm equation (4) for the Langmuir model are ${ }^{32,33}$ :

$\Pi=-\frac{R T}{\omega} \ln (1-\Gamma \omega)$, 
$b c=\frac{\Gamma \omega}{1-\Gamma \omega}$

where $\Pi$ is the surface (interfacial) pressure, $R$ the gas law constant, $T$ the absolute temperature, $\omega$ the molar area, $\Gamma$ the interfacial excess concentration of the surfactant, and $c$ the surfactant bulk concentration. The isotherm equation (4) can be interpreted using a kinetic model that considers the adsorption and desorption fluxes during the formation of the interfacial layer

$\frac{d \Gamma}{d t}=k_{\mathrm{ad}} c\left(1-\frac{\Gamma}{\Gamma_{\infty}}\right)-k_{\mathrm{des}} \frac{\Gamma}{\Gamma_{\infty}}$

where $k_{\mathrm{ad}}$ and $k_{\mathrm{des}}$ are the rate constants of adsorption and desorption of the surfactant, respectively, $\Gamma_{\infty}$ the limiting adsorption, and $\Gamma_{\infty}=1 / \omega$. At equilibrium $\mathrm{d} \Gamma / \mathrm{d} t=0$, then Eq. (5) transforms into Eq. (4) with $b=k_{\text {ad }} / k_{\text {des. }}$.

\section{Frumkin Model}

The adsorption isotherm (6) and the equation of state (7) for adsorption layers proposed by Frumkin ${ }^{34}$ which describe the adsorption of low-molecular-weight surfactants when the systems deviate from an ideal (Langmuir) behavior by the intermolecular interaction parameter, $a$ :

$-\frac{\Pi \omega}{R T}=\ln (1-\Gamma \omega)+a(\Gamma \omega)^{2}$

$b c=\frac{\Gamma \omega}{1-\Gamma \omega} \exp (-2 a \Gamma \omega)$

\section{Proteins}

The thermodynamic model derived by Fainerman et al. 35-38 assumes that adsorbed proteins can exist in different states in the interfacial layer, requiring a molar area varying from a maximum value $\left(\omega_{\max }\right)$ at very low surface coverage to a minimum value $\left(\omega_{\min }\right)$ at high surface coverage. The molar areas of two "neighbouring" conformations differ from each other by the increment $\omega_{0}$, chosen to be equal to the molar area of the solvent, or the area occupied by one segment of the adsorbed protein molecule. The resulting equation of state, accounting for non-ideal entropy and enthalpy of mixing for the surface layer reads:

$-\frac{\Pi \omega_{0}}{R T}=\ln \left(1-\Theta_{\mathrm{P}}\right)+\Theta_{\mathrm{P}}\left(1-\frac{\omega_{0}}{\omega}\right)+a_{\mathrm{P}} \Theta_{\mathrm{P}}^{2}$

Here $\Pi$ is the surface pressure, $R$ the gas law constant, $T$ the temperature, $\omega$ the average molar area of the protein, $\alpha_{\mathrm{p}}$ is the intermolecular interaction parameter, $\theta_{\mathrm{P}}=\omega \Gamma=$ $\sum_{i=1}^{n} \omega_{i} \Gamma_{i}$ the total surface coverage by protein, and $\Gamma_{\mathrm{i}}$ the protein adsorption in the $i$ th state.
The total protein adsorption is given by

$\Gamma=\sum_{i=1}^{n} \Gamma_{i}$

The adsorption isotherm for each state $(i)$ of the adsorbed protein reads

$b_{\mathrm{P}} c_{\mathrm{P}}=\frac{\omega \Gamma_{\mathrm{i}}}{\left(1-\Theta_{\mathrm{P}}\right)^{\omega_{\mathrm{i}} / \omega}} \exp \left[-2 a_{\mathrm{P}}\left(\frac{\omega_{\mathrm{i}}}{\omega}\right) \Theta_{\mathrm{P}}\right]$

where $c_{\mathrm{P}}$ is the protein bulk concentration and $b_{\mathrm{P}}=b_{\mathrm{i}}$ the equilibrium adsorption constant for the protein in the $i$ th state. According to Fainerman et al. ${ }^{38}$, it was assumed that for all states the constants $b_{\mathrm{i}}$ have the same value, and therefore the adsorption constant for the protein molecule as a whole is $\Sigma b_{\mathrm{i}}=n b_{\mathrm{i}}$. The set of Eqs. (8,9 and 10) describes the evolution of protein adsorption states during the formation of an adsorption layer.

Mixtures of Proteins and Low-molecular-weight

Surfactants

Mixed adsorption of proteins and low-molecular-weight surfactants have been described recently by a quantitative thermodynamic model ${ }^{22,23}$. The main equations describing equilibrium surface layers, as well as the adsorption kinetics, have been summarized in detail before ${ }^{22,23,39}$. The software for data analysis can be downloaded from the internet ${ }^{40}$.

The following mathematical models are applied for the non-ideal behavior of a system which contains a single component able to exist in different states $^{32}$.

Equation of state:

$$
\begin{gathered}
-\frac{\Pi \omega_{0}^{*}}{R T}=\ln \left(1-\theta_{\mathrm{P}}-\theta_{\mathrm{S}}\right)+\theta_{\mathrm{P}}\left(1-\omega_{0} / \omega_{\mathrm{P}}\right) \\
+a_{\mathrm{P}} \theta_{\mathrm{P}}^{2}+a_{\mathrm{S}} \theta_{\mathrm{S}}^{2}+2 a_{\mathrm{PS}} \theta_{\mathrm{P}} \theta_{\mathrm{S}}
\end{gathered}
$$

Where $\Pi$ is the interfacial pressure, $R$ the gas law constant, $T$ the absolute temperature and

$\omega_{0}^{*}=\frac{\omega_{0} \theta_{\mathrm{P}}+\omega_{\mathrm{S}} \theta_{\mathrm{S}}}{\theta_{\mathrm{P}}+\theta_{\mathrm{S}}}$

Here $\theta_{\mathrm{p}}$ is the surface coverage by protein molecules:

$\theta_{\mathrm{P}}=\omega \Gamma=\sum_{i=1}^{n} \omega_{i} \Gamma_{i}$

where $\Gamma_{i}$ is the protein adsorption in the $i$ th state, and $\omega$ the effective area per protein molecule;

$\Gamma=\sum_{i=1}^{n} \Gamma_{i}$ 
is the total adsorption of the protein; and

$\theta_{\mathrm{S}}=\omega_{\mathrm{S}} \Gamma_{\mathrm{S}}$

is the surface coverage by low-molecular-weight surfactant and $\Gamma_{\mathrm{S}}$ is the corresponding adsorption.

$\alpha_{\mathrm{PS}}$ corresponds to the Frumkin parameter for the interaction between the protein and the low-molecularweight surfactant

$\alpha_{\mathrm{S}}$ is the Frumkin parameter for the interaction between the surfactant molecules

$\alpha_{\mathrm{P}}$ is the Frumkin parameter for the interaction between the protein molecules

The adsorption isotherm for the protein in the $i$ th state in the presence of low-molecular-weight surfactant can be expressed as:

$b_{\mathrm{P}} c_{\mathrm{P}}=\frac{\omega \Gamma_{i}}{\left(1-\theta_{S}-\theta_{P}\right)^{\omega_{i} / \omega}} \exp \left[-2 a_{\mathrm{P}}\left(\omega_{i} / \omega\right) \theta_{\mathrm{P}}-2 a_{\mathrm{PS}} \theta_{\mathrm{S}}\right]$

where $c_{\mathrm{P}}$ is the protein concentration in the surface layer and $b_{\mathrm{P}}$ the adsorption constant of the protein at equilibrium.

The adsorption isotherm of the surfactant in the presence of protein can be written:

$b_{\mathrm{S}} c_{\mathrm{S}}=\frac{\theta_{\mathrm{S}}}{\left(1-\theta_{\mathrm{S}}-\theta_{\mathrm{P}}\right)} \exp \left[-2 a_{\mathrm{S}} \theta_{\mathrm{S}} 2 a_{\mathrm{PS}} \theta_{\mathrm{P}}\right]$

where $c_{\mathrm{S}}$ is the low-molecular-weight surfactant concentration in the surface layer and $b_{\mathrm{S}}$ the adsorption constant of the lowmolecular-weight surfactant at equilibrium.

The relation between the adsorption of protein and of low-molecular-weight surfactant can be derived from the adsorption isotherm given by Eqs. (18) and (19)

$\frac{\Gamma_{\mathrm{P}} \omega_{\mathrm{P}}}{\Gamma_{\mathrm{S}} \varpi_{\mathrm{S}}}=\frac{b_{\mathrm{P}} c_{\mathrm{P}}}{b_{\mathrm{S}} c_{\mathrm{S}}}\left(1-\Gamma_{\Sigma} \omega\right)^{\frac{\omega_{\mathrm{P}}-\omega_{\mathrm{S}}}{\omega}}$

where

$\Gamma_{\Sigma}=\Gamma_{1}+\Gamma_{2}$

The average molar area of the adsorbed molecules can be written:

$\omega=\frac{\Gamma_{P} \omega_{P}+\Gamma_{S} \varpi_{S}}{\Gamma_{P}+\Gamma_{S}}$

For $\omega_{\mathrm{P}} \gg \omega_{\mathrm{S}}$ at a given ratio of the concentrations in the bulk solution, the portion of protein in the surface layer decreases sharply with the increase of the total adsorption $\Gamma_{\Sigma}$.

\section{Results}

The effect of Sn-2 MC16 on the interfacial tension of a buffer/decane system previously adsorbed with pancreatic lipase was studied. Figure 2 shows that the monoglyceride is able to decrease the interfacial tension to values close to the pure monoglyceride.

With tensiometry, one follows the change in surface free energy but the technique does not per se give information about what molecules are present at the interface. Interfacial shear rheology gives complementary information because it can distinguish between low and high molecular weight amphiphilic compounds in the interfacial layer. The torsion pendulum technique, which has been used in this work, gives information about the stiffness of the interfacial layer. By measuring the variation of oscillation amplitude with time, a change in rheology properties of this layer can be detected.

Figure 3a shows the response to shear deformation of the phosphate buffer/decane interface covered with lipase from porcine pancreas at a concentration corresponding to full surface coverage. Measurements were made immediately after formation of the interface, after $30 \mathrm{~min}$ rest and after $60 \mathrm{~min}$ rest. With the crude lipase preparation at the interface there is some initial oscillation but it is rapidly damped. The experiment, after 60 -min rest, shows lower oscillation amplitude as a result of an increased stiffness of the interfacial layer. In a recent publication ${ }^{41}$, it was shown that lipase from $R$. miehei behaves like a small-molecularweight surfactant at the interface (even when the interface is saturated with the enzyme). Therefore, the high stiffness verified by ISR for the crude preparation of pancreatic lipase should be either attributed to the presence of

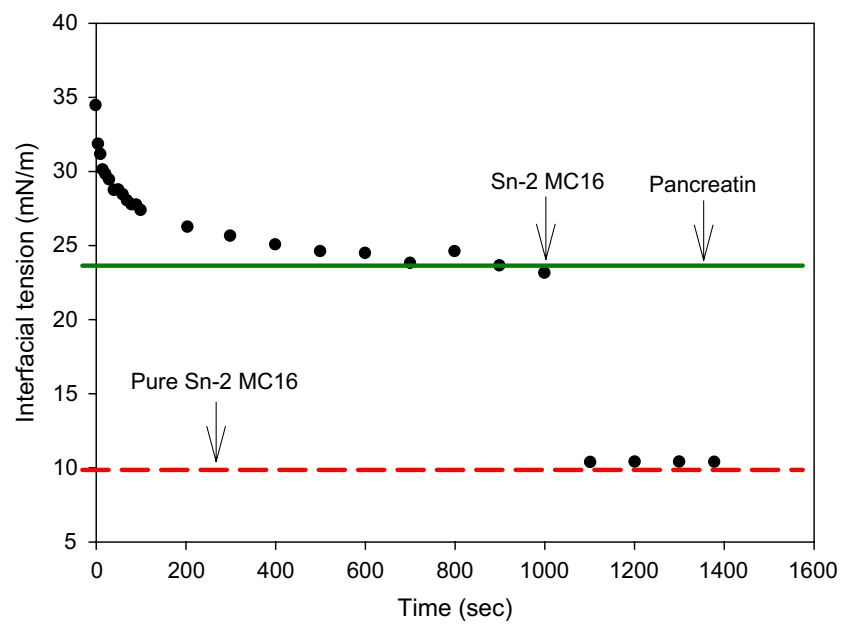

Fig. 2 Interfacial tension of a buffer/decane system with $10 \mathrm{mg} / \mathrm{ml}$ of a crude solution with pancreatic lipase and further injection into the oil phase of $6.7 \times 10^{-4} \mathrm{M}$ of Sn-2 monopalmitin (Sn-2 MC16) after $1,000 \mathrm{~s}$ of lipase adsorption 

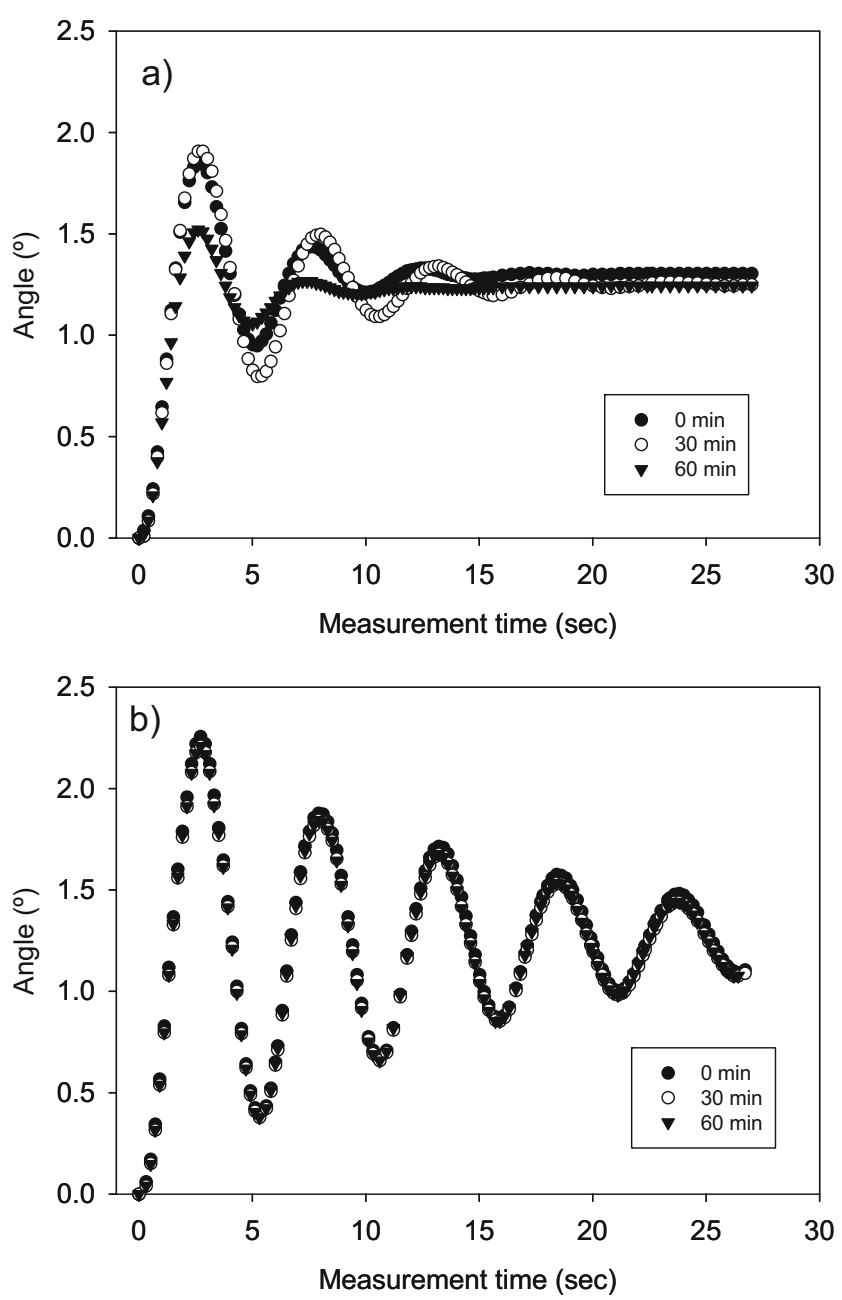

Fig. 3 Variation of the torsion angle with time after formation of a phosphate buffer/decane interface with a solution of $10 \mathrm{mg} / \mathrm{ml}$ lipase from porcine pancreas as water phase without (a) and with (b) $6.6 \times$ $10^{-4} \mathrm{M} \mathrm{Sn}-2$ monopalmitin solution

contaminant proteins or due to the colipase effect in anchoring the lipase at the interface. Nevertheless, the purpose of using pancreatin instead of pure enzymatic forms is to assess the interfacial behavior of physiological relevant solutions and its interaction with surfactants. Figure $3 \mathrm{~b}$ shows the corresponding curves for addition of Sn-2 MC16 added to the same two-phase system with the Sn-1,3 regiospecific lipase from porcine pancreas covering the interface. The damping pattern is identical to that of the pure monoglyceride (data not shown). The result is a further proof of the Sn-2 monoglyceride rapidly replacing the lipase from the interface. The situation at the interface is stable during the time of the experiment, $60 \mathrm{~min}$, as judged from the identical damping curves.

Figure 4 shows the interfacial coverage as a function of Sn-2 MC16 bulk concentration for a fixed concentration of either $3.5 \times 10^{-5}$ or $5.0 \times 10^{-3} \mathrm{M}$ of lipase from $R$. miehei and TC8, respectively. The results were obtained by mathematical modeling after having experimentally determined the interfacial area and adsorption constant of each molecule from the adsorption isotherms ${ }^{41,42}$. Moreover, the interfacial coverage of a phosphate buffer/decane system saturated with lipase was calculated for increasing bulk concentrations of Sn-2 MC16. As can be seen, lipase, and TC 8 can easily be expelled from the interface by the more interfacial active monoglyceride. In fact, the concentration of monoglyceride needed to desorb $50 \%$ of TC8 from the interface is about two orders of magnitude lower than the one required for desorbing the lipase. The lipase concentration used corresponds to saturation of the buffer-decane interface in absence of other interfacially active molecules. Since under physiological conditions lipase is always present at much lower concentration, the results indicate that a Sn-2 monoglyceride can completely desorb the lipase from the interface.

Figure 5 shows results obtained in the TIM model concerning the effect of three different interfacially active molecules on the lipolytic generation of caprylic acid from tricaprylin in the lumen of the stomach, duodenum, and jejunum. While in the stomach all the tested surfactants have an inhibitory effect on the lipolysis, only Sn-2 MC16 has the same regulatory effect in the duodenum and the jejunum. In fact, our data suggest that LysoPC and BLG have a stimulatory effect on triglyceride hydrolysis after the gastric phase.

Figure 6 shows that the Sn-2 MC16 decreased the concentration of caprylic acid in the efflux throughout the digestion period. This result confirms that less TC8 is hydrolyzed by both gastric and pancreatic lipase when

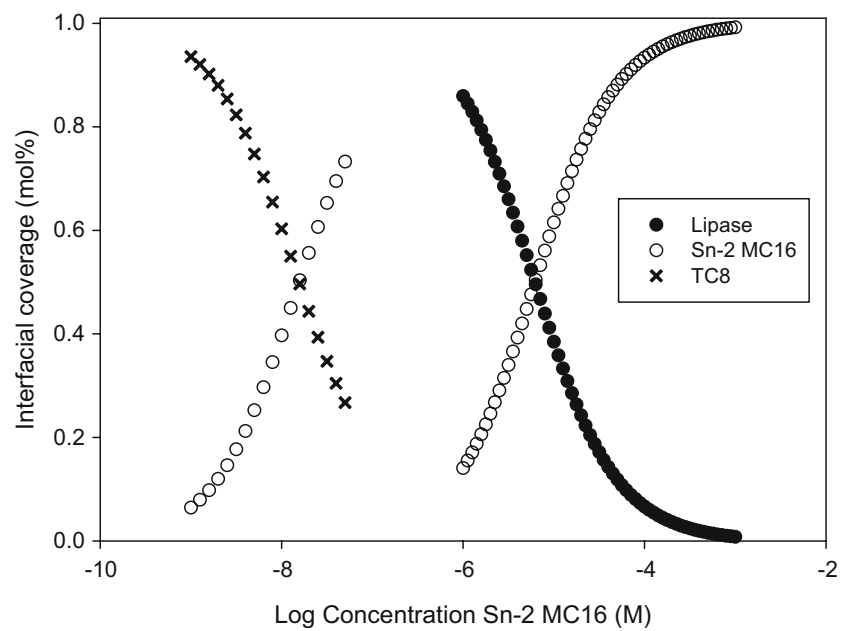

Fig. 4 Interfacial coverage as a function of bulk concentration of Sn-2 monopalmitin $(\mathrm{Sn}-2 \mathrm{MClO})$ for a fixed concentration $\left(3.5 \times 10^{-5} \mathrm{M}\right)$ of lipase from $R$. miehei or for a fixed concentration $\left(5.0 \times 10^{-3} \mathrm{M}\right)$ of tricaprylin $(T C 8)$ 
a)

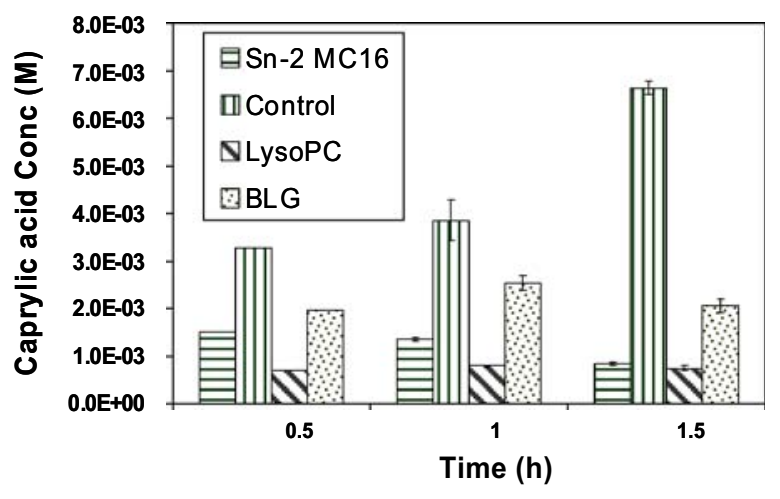

b)

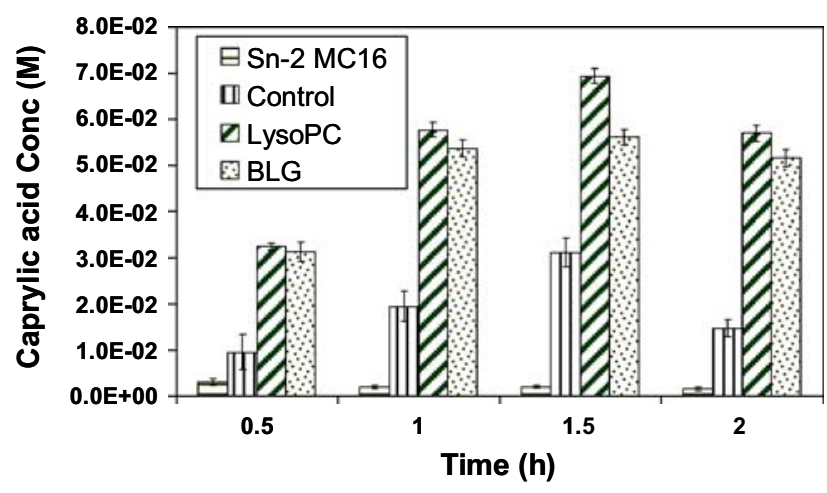

c)

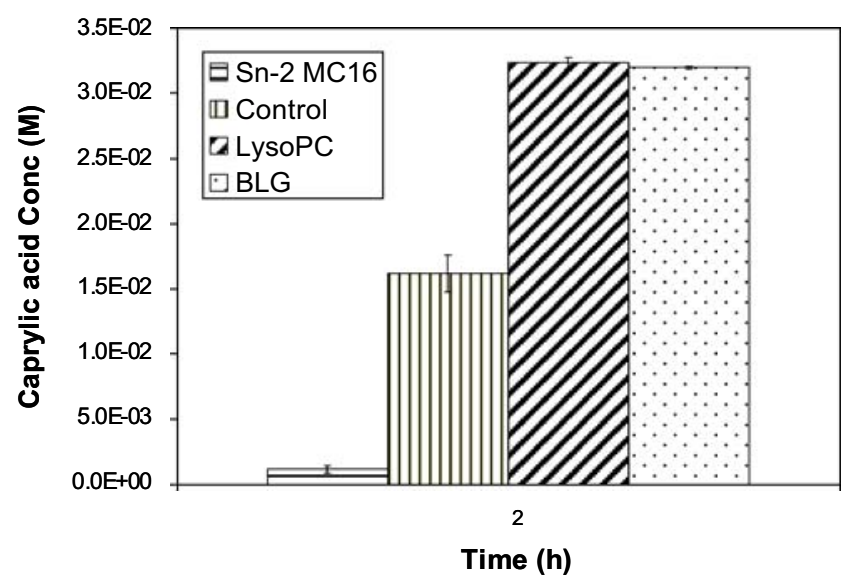

Fig. 5 Caprylic acid concentrations obtained in the lumen of stomach (a), duodenum (b), and jejunum (c), after digestion of tricaprylin in a surfactant-free system (control), and in the presence of Sn-2 monopalmitin (Sn-2 MC16), lysophosphatidylcholine (LysoPC) or $\beta$ lactoglobulin $(B L G)$

monoglyceride is present. On the contrary, both the presence of LysoPC and BLG increased the degree of hydrolysis as compared to the control which does not contain an amphiphile.

Figure 7 is coherent with the previous results. It shows that the presence of Sn-2 MC16 strongly reduces the amount of caprylic acid after absorption from both the ileum and the jejunum.

Figure 8 shows a GC-FID chromatogram of an aliquot corresponding to the absorption in the ileum during the first $2 \mathrm{~h}$ of digestion of TC8. The two chromatograms constitute further evidence that the presence of Sn-2 MC16 decreases the rate of hydrolysis of TC8.

\section{Discussion}

The previously discussed results show that both lipase and triglyceride are efficiently displaced from the oil-water interface by the more interfacially active $\mathrm{Sn}-2$ monoglyceride. It was also found that pancreatic lipase with colipase could be replaced from a buffer-decane interface by Sn-2 MC16. The latter observation confirms the previously expressed view that the anchoring effect of colipase has its limitations ${ }^{18}$.

Frazer et al $^{43}$ found that bile salts provide an effective emulsifying system for triglycerides and compounds of similar polarity. However, bile has been shown to enhance the digestion only under certain very limited conditions; otherwise, bile salts have no or even an inhibitory effect on lipolysis ${ }^{44}$. According to Miller and Small ${ }^{45}, 2.5$ to $4 \%$ of emulsified triglyceride is present at the oil-water interface when bile is used as emulsifier.

One may anticipate that the rate of lipase-catalyzed hydrolysis of triglycerides in an oil-water system will be slow if the interface is covered by an amphiphile that is resistant to enzymatic hydrolysis. Our results show that concentrations as low as $2.810^{-8} \mathrm{M}(\mathrm{mol} / \mathrm{mol})$ of mono-

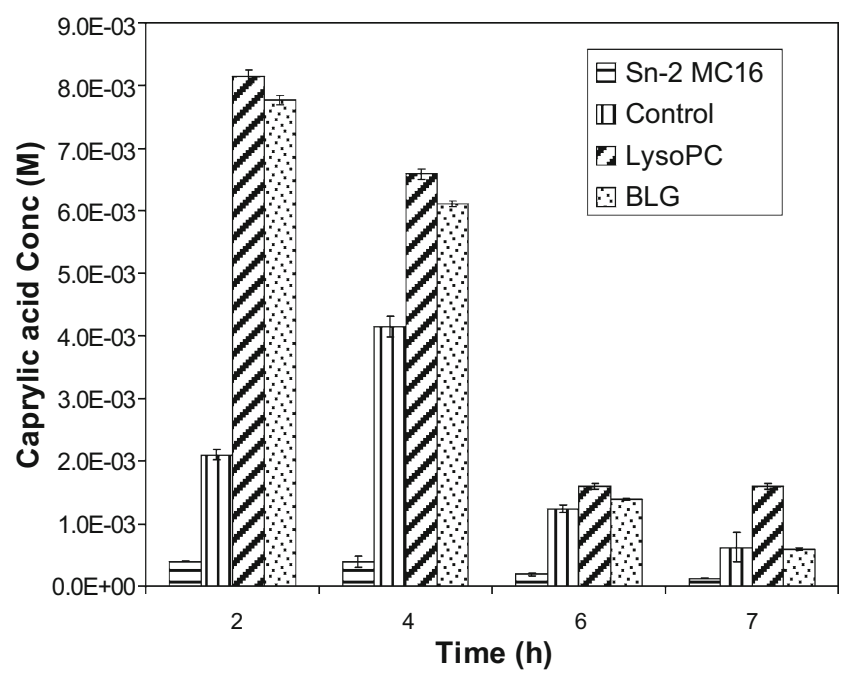

Fig. 6 Caprylic acid concentrations obtained in the efflux after digestion of tricaprylin in a surfactant-free system (control), and in the presence of Sn-2 monopalmitin ( $\mathrm{Sn}-2 \mathrm{MC16})$, lysophosphatidylcholine $(L y s o P C)$ or $\beta$-lactoglobulin $(B L G)$ 
a)

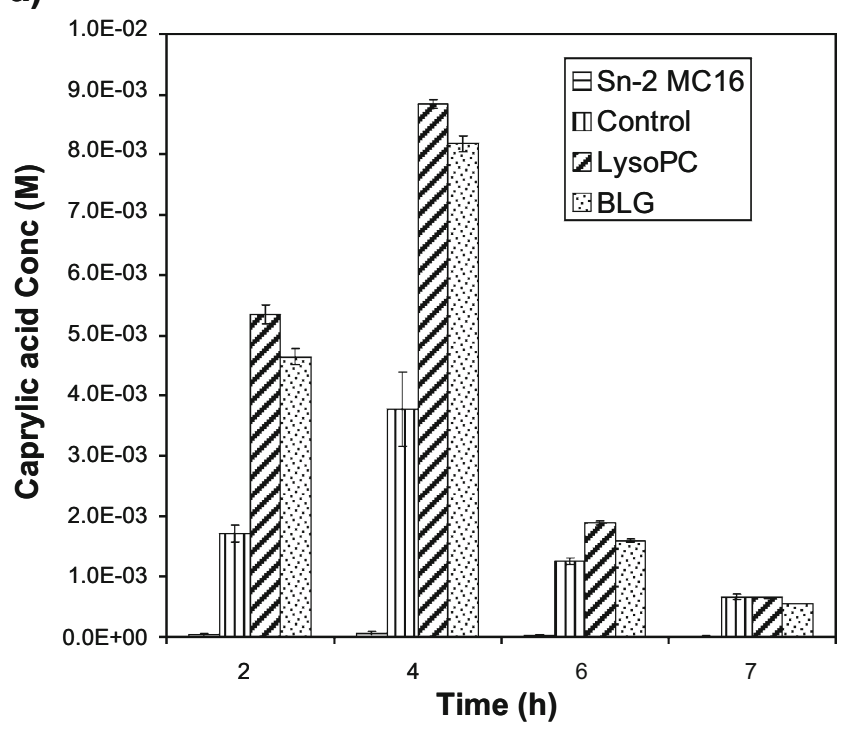

b)

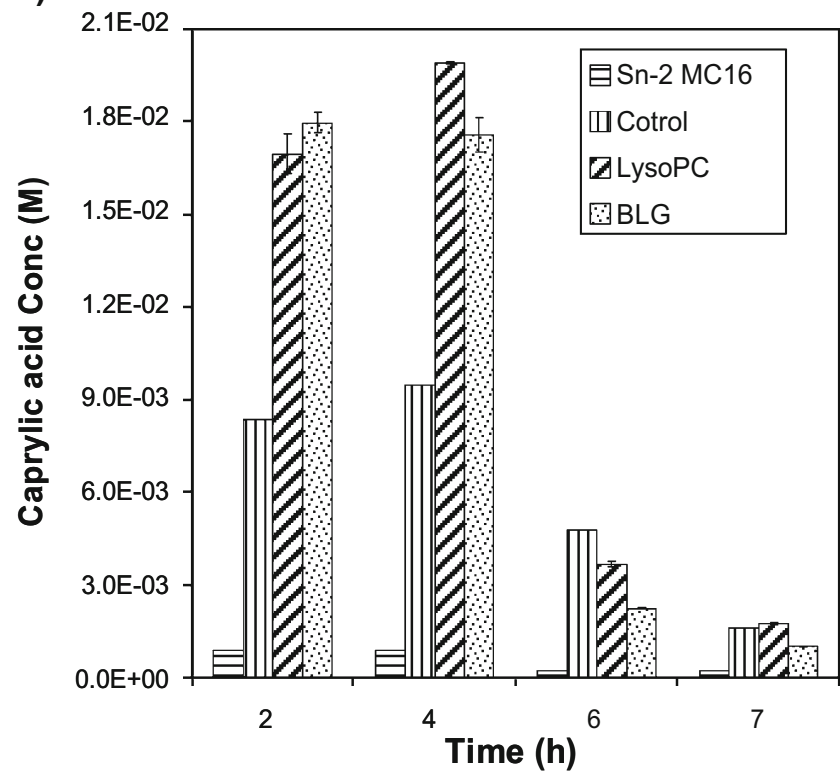

Fig. 7 Caprylic acid concentration obtained upon absorption in the ileum (a) and the jejunum (b) after digestion of tricaprylin in a surfactant-free system (control), in the presence of Sn-2 monopalmitin (Sn-2 MC16), lysophosphatidylcholine (LysoPC) or $\beta$-lactoglobulin $(B L G)$

glyceride in the bulk phase can displace $50 \%(\mathrm{~mol} / \mathrm{mol})$ of triglyceride. On the contrary, the desorption of the lipase is more difficult since more Sn-2 monoglycerides, i.e., 8.0 $10^{-6} \mathrm{M}$, is required to displace $50 \%$ of the enzyme from the interface. These desorption phenomena have a clear impact on the fat digestion as shown in the presented TIM experiments.

Figure 9 illustrates our view on how a Sn-2 monoglyceride expels both the triglyceride and the lipase from the oil-water interface. We have previously shown that the monoglycerides are the most interfacially active molecules generated during lipolysis ${ }^{41,42,46}$. As an example, the interfacial tensions for a decane-buffer interface saturated with dicaprylin, monocaprylin, caprylic acid, and $R$. miehei lipase were $22 ; 4,25$, and $15 \mathrm{mN} / \mathrm{m}$ at neutral $\mathrm{pH}^{46}$. Separation of a Sn-1,3 regiospecific lipase from the substrate, the triglyceride, by a monolayer of a Sn-2 monoglyceride formed at the water-oil interface is an obviously efficient way of preventing further fat digestion, as is shown in the experiments with the model gastro-intestinal system.

Let us now briefly discuss the impact of bile salt micelles in the digestion of fat. One should take into consideration that solubilization of interfacially active molecules by bile is only possible in the duodenum. However, lipolysis starts earlier in the stomach, where the concentration of amphiphilic compounds is low. Actually, the TIM experiments show that gastric lipolysis is reduced in the presence of all the tested interfacially active molecules. This result can be explained with a reduced accessibility of gastric lipase for the triglyceride. However, an increased lipolysis in all the other compartments of the gastro-intestinal tract is found for the tested meals enriched with either LysoPC or BLG. It is very likely that this is due to an increased interfacial area leading to an enhanced exposure of triglyceride to the lipase. Among the three different amphiphiles tested for controlling lipolysis, only BLG can be cleaved during digestion. BLG is known to be a substrate for proteases. The other two amphiphiles, Sn-2 MC16 and LysoPC, are not cleavable by lipases or phospholipases. In the duodenum, there is a pronounced trypsin-catalyzed proteolysis. BLG is converted into peptides, which are not very surface active and which allow the lipase to access the substrate without major restrictions.

Previous reports ${ }^{47}$ have described that LysoPC causes an increase in the $K_{\mathrm{m}}$ and a decrease in $V_{\max }$ values for the interaction between pancreatic lipase and triacylglycerol. This indicates that the substrate affinity and catalytic activity is inhibited by LysoPC. Our results show that gastric lipolysis of TC8 is decreased by LysoPC. This regulatory effect can most likely be attributed to a decreased substrate accessibility to the enzyme. LysoPC, which is naturally formed from the hydrolysis of phosphatidylcholine by phospholipase $\mathrm{A}_{2}$, is more interfacially active than lipase or TC8 (data not shown). Therefore, at the model stomach environment, the interface will be depleted from triglyceride and preferentially occupied by the lysophospholipid.

However, in the duodenum, lysophospholipids will be most likely solubilized into bile salts micelles. Actually, the addition of taurodeoxycholic acid sodium salts or phospholipids was shown to restore the inhibitory effect of lysophospholipids on pancreatic lipase ${ }^{47}$. In the present work, we have observed an increased hydrolysis of TC8 in 
Fig. 8 Chromatograms obtained from ileum absorption after $2 \mathrm{~h}$ digestion of tricaprylin in TIM $\mathbf{a}$ in the presence of Sn-2 monopalmitin, b without Sn-2 monopalmitin (control) a)

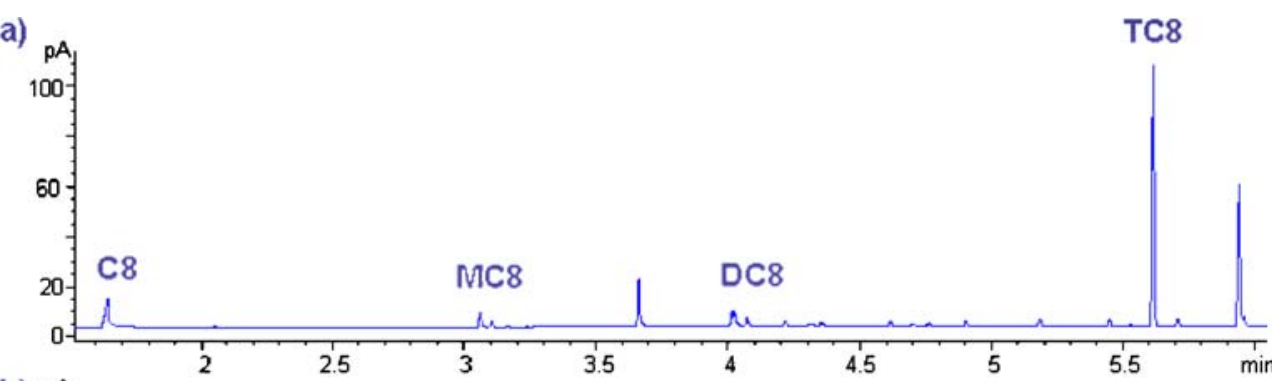

b)

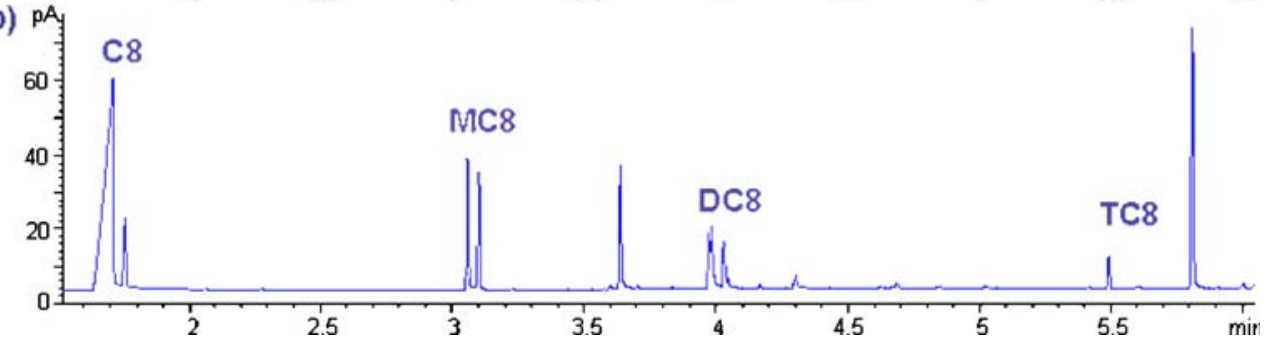

the duodenum as compared to the control. We attribute this result to an increased interfacial area induced by LysoPC at micellar concentrations where substrate accessibility to lipases is not compromised.

Addition of a Sn-2 monoglyceride resulted in a strong reduction of the amount of caprylic acid generated

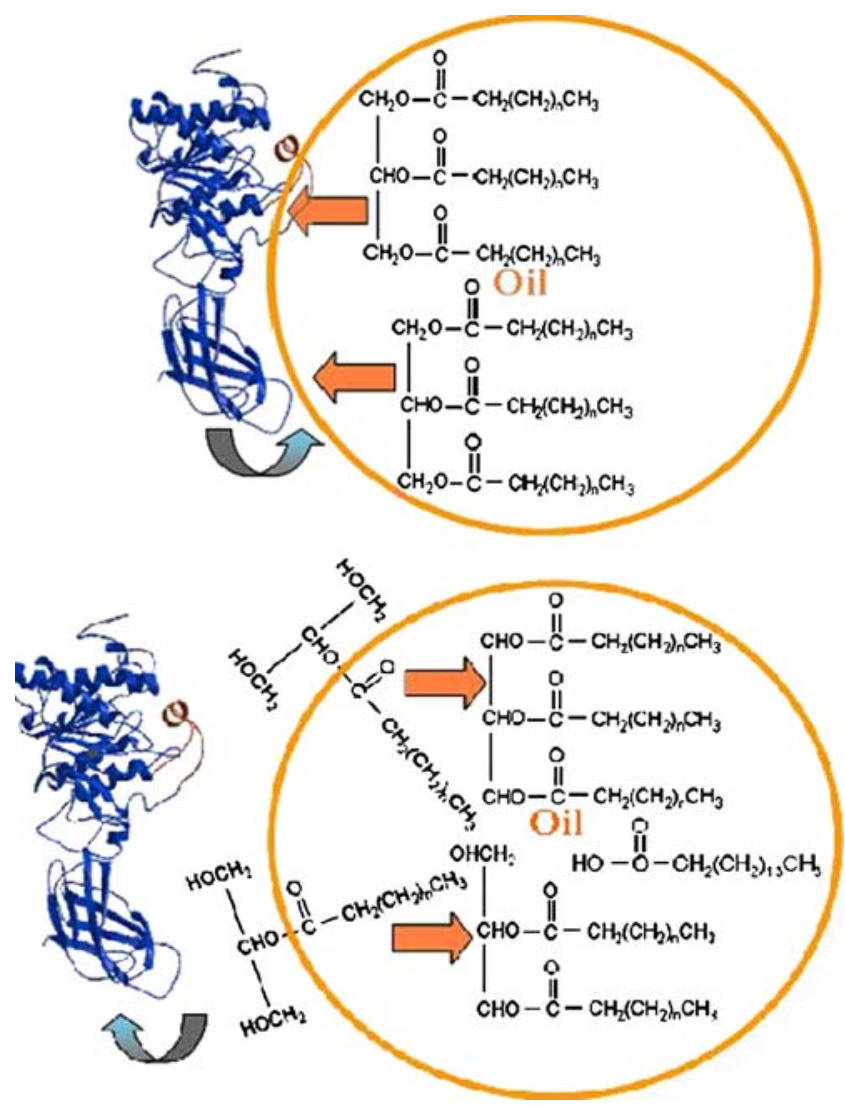

Fig. 9 Generated non-cleavable surface-active Sn-2 monoglycerides exclude triglycerides from the interface and push the lipase into the sub-layer throughout the model gastro-intestinal system. The reduced exposure of the triglyceride for the lipase as an effect of the lipase-resistant monoglyceride dominating the interface explains this effect. Contrary to LysoPC, Sn-2 monoglycerides most likely did not allow substrate accessibility to lipase even in the presence of bile. Due to the solubility properties of the tested surfactants, it is reasonable to hypothesize that $\mathrm{Sn}-2$ monoglycerides are the least solubilized into bile salt micelles. Therefore, an accumulation of Sn-2 monoglycerides at the water-oil interface is expected to have a strong influence on lipolysis.

The publication by Mun et al. ${ }^{48}$ compares the effect of different emulsifiers (whey protein isolate, sodium caseinate, lecithin, and tween-20) on in vitro digestibility of lipid droplets catalyzed by pancreatic lipase. It is claimed that the interfacial layer surrounding the lipid droplets play an important role in determining the rate and extent of lipid hydrolysis. However, some key interfacial properties of the tested surfactants are not assessed (e.g., adsorption rate constant, molecular area, interaction parameter, compressibility). Therefore, with this lack of information it is impossible to do a precise characterization of the molecules that populate the interface in complex systems (e.g., determine interfacial coverage of each molecule at the interface). It is likely that proteins and ionic surfactants, which are normally not extremely interfacially active, allow the co-presence of triglycerides at the interface. On the other hand, non-ionic low-molecular-weight surfactants pack very tightly at the interface and the co-presence of triglycerides at the interface is much less likely. Moreover, in a previous communication ${ }^{41}$, we have shown that lipases are more interfacially active than other common globular proteins. Therefore, lipase may prevail at the interface even when there is a large excess of other proteins in the bulk solution (e.g., whey protein and sodium caseinate). 
In in vivo systems, the in situ generated Sn-2 monoglycerides are solubilised by bile salts from the interface of water/oil drops and transported across the brush border cells of the gastro-intestinal system. This delivery mechanism provides a reestablishment of the solubility capacity of bile salts and a high yield of fat absorption. This process does not occur in the adopted gastro-intestinal system and therefore bile salt remains saturated with Sn-2 monoglycerides. Therefore, the accumulation of this reaction product at the interface controls the lipolysis. It has been reported that at least during the initial stages of fat digestion, the rate of lipolysis significantly exceeds the capacity to incorporate the resulting fatty acids and monoglycerides into mixed bile salt micelles ${ }^{49}$. Therefore these polar lipids accumulate at their site of origin, the oilwater interface, a self-regulation mechanism. Consequently, the high efficiency of fat digestion in in vivo systems is attributed to the transport mechanisms of interfacially active molecules from the interface of water/oil drops and further delivery of these molecules at the brush border cells. Any mechanism that affects bile salt solubility (e.g., ionic strength) should then affect the extension of fat digestion due to accumulation of non-cleavable interfacially active molecules at the water/oil interface.

\section{Concluding Remarks}

The effect of interfacial active molecules on the digestion of fat was studied in a model gastro-intestinal system. We show that gastric lipolysis is very sensitive to the presence of surfactants and that lipolysis of triglycerides is decreased in the presence of Sn-2 MC16, BLG, or LysoPC. This effect is attributed to substrate interfacial depletion by the more amphiphilic molecules. Our adopted mathematical model confirms that TC8 is replaced from the interface at low concentrations of monoglyceride.

In the duodenum, the hydrolysis and solubilization of amphiphiles into bile salt micelles boosts lipolysis of triglycerides. This phenomenon is most likely crucial for lipase substrate accessibility and further extension of triglyceride hydrolysis. Interestingly enough, Sn-2 monopalmitin was shown to have a regulatory effect of lipolysis even in the presence of bile.

From our results, it is reasonable to conclude that the accumulation of highly interfacial active molecules in the lumen of the gastro-intestinal tract (e.g., due to problems of absorption), have a regulatory effect on the lipolysis. We anticipate that our findings provide a new approach for the control of fat metabolism which is central to health threats like obesity and diabetes mellitus $\mathrm{II}^{50}$ and important for the regulation of energy metabolism in general ${ }^{51}$.

\section{References}

1. D.J. McClements, Food Emulsions: Principles, Practise, and Techniques (CRC, Boca Raton, 2004)

2. D. Lairon, Eur. J. Clin. Nutr. 50, 125 (1996)

3. G.A. Van Aken, in Coalescence mechanisms in protein-stabilized emulsions, ed. by S. Friberg, K. Larsson, J. Sjoblom. Food Emulsions, 4th edth edn. (Marcel Dekker, New York, NY, 2004)

4. S. Mun, E. Decker, Y. Park, J. Weiss, D.J. McClements, FOBI 1, 21 (2006)

5. W. Pafumi, D. Lairon, P. Porte, C. Juhel, J. Storch, M. Hamosh, M. Armand, JBC. 31, 28070 (2002)

6. M.C. Carey, D.M. Small, C.M. Bliss, Annu. Rev. Physiol. 45, 651 (1983)

7. M. Hamosh, D. Ganot, P. Hamosh, J. Biol. Chem. 254, 12121 (1979)

8. L. Van Gaal, I. Mertens, D. Ballaux, Best Pract Res. Clin. Gastroenterol. 18, 1049 (2004)

9. R. Verger, TIBTECH. 15, 32 (1997)

10. M. Wickham, M. Garrood, J. Leney, P. Wilson, A. Fillery-Travis, J. Lipid Res. 39, 623 (1998)

11. H. Brockman, W. Momsen, T. Tsujita, JAOCS 65, 891 (1988)

12. P. Skagerlin, B. Folmer, B.K. Jha, M. Svensson, K. Holmberg, Colloid Polym. Sci. 108, 47 (1998)

13. P. Canioni, R. Julien, J. Rathelot, L. Sarda, Biochimie. 58, 751 (1976)

14. T. Kawase, T. Hashimoto, T. Fujii, M. Minagawa, Yukagaku. 34, 530 (1985)

15. P. Skagerlind, M. Jansson, B. Bergenstahl, K. Hult, Colloids Surf B. 4, 129 (1995)

16. J.S. Patton, M.C. Carey, Am J Physiol Gastrointest. Liver Physiol. 241, G328 (1981)

17. A. Tiss, F. Carriere, R. Verger, Anal. Biochem. 294, 36 (2001)

18. Y. Gargouri, R. Julien, A.G. Bois, R. Verger, L. Sarda, J. Lipid Res. 24, 1336 (1983)

19. M. Minekus, M. Jelier, J. Xiao, S. Kondo, K. Iwatsuki, S. Kokubo, M. Bos, B. Dunnewind, R. Havernaar, Biosci. Biotechnol. Biochem. 69, 932 (2005)

20. S. Bellmann, R. Havenaar, Ernährungs. Umschau. 58, 450 (2007)

21. V.B. Fainerman, S.A. Zholob, M. Leser, M. Michel, R. Miller, J Colloid Interface Sci. 274, 496 (2004)

22. R. Miller, V.B. Fainerman, M. Leser, M. Michel, Curr Opin Colloid Interface Sci. 9, 350 (2004)

23. R. Miller, D.O. Grigoriev, E.V. Aksenenko, S.A. Zholob, M. Leser, M. Michel, V.B. Fainerman, in Thermodynamics and adsorption kinetics studies of proteins + surfactants mixtures, ed. by E. Dickinson. Food Colloids: Interactions, Microstructure and Processing (Royal Society of Chemistry, Special Publ, 2005)

24. E.H. Lucassen-Reynders, Colloids Surf A 91, 79 (1994)

25. M. Minekus, P. Marteau, R. Havenaar, J. Huis in 't Veld, ATLA 23, 197 (1995)

26. M. Minekus, R. Havenaar, United States Patent nr. 5,525,305 (1996).

27. M. Minekus, J. Lelieveld, H. van den Berg, A dynamic model of the stomach and small intestine to study the bioaccessibility of carotenoids from vegetables and the effect of processing. In: Abstract Book of the Bioavailability Congress, Interlaken, Switzerland, p. 111, 2001.

28. S. Southon, Model systems in vitro and in vivo, for predicting the bioavailability of lipid soluble components in food. FAIR CT973100 (Final Report), 30-4-0001, 2001.

29. J.D. Elashoff, T.J. Reedy, J.H. Meyer, Gastroenterology 83, 1306 (1982)

30. G. Loglio, P. Pandolfini, R. Miller, A.V. Makievski, F. Ravera, M. Ferrari, L. Liggieri, in Novel methods to study interfacial layers, 
ed. by D. Möbius, R. Miller. Studies in Interface Science (Elsevier, Amsterdam, 2001)

31. J. Krägel, S. Siegel, R. Miller, M. Born, K. Schano, Colloids Surf A 91, 169 (1994)

32. V.B. Fainerman, R. Miller, in Surfactants - Chemistry, Interfacial Properties and Application, Studies, ed. by V.B. Fainerman, D. Möbius, R. Miller. Interface Science Vol. 13 (Elsevier, Amsterdam, 2001)

33. I. Langmuir, J. Amer. Chem. Soc. 39, 1848 (1917)

34. A.N.Z. Frumkin, Phys. Chem. 116, 466 (1925)

35. A.V. Makievski, V.B. Fainerman, M. Bree, R. Wüstneck, J. Krägel, R. Miller, J. Phys. Chem. 102, 417 (1998)

36. V.B. Fainerman, R. Miller, R. Wüstneck, J. Colloid Interface Sci. 183, 25 (1996)

37. V.B. Fainerman, R. Miller, Langmuir 15, 1812 (1999)

38. V.B. Fainerman, E.H. Lucassen-Reynders, R. Miller, Adv. Colloid Interface Sci. 16, 237 (2003)

39. V.B. Fainerman, S.A. Zholob, M. Leser, M. Michel, R. Miller, J. Phys. Chem. 108, 16780 (2004)

40. Access to software packages is free of charge via http://www. sinterface.com or http://www.mpikg.mpg.de/gf/miller/.
41. P. Reis, R. Miller, J. Krägel, M.E. Leser, V.B. Fainerman, H. Watzke, K. Holmberg, Lipases at interfaces: Unique interfacial properties as globular proteins, Langmuir (2008), in print

42. P. Reis, R. Miller, M.E. Leser, H. Watzke, V.B. Fainerman, K. Holmberg, Adsorption of polar lipids at the water-oil interface, Langmuir (2008), in print.

43. A.C. Frazer, J.H. Schulman, H.C. Stewart, J. Physiol. 103, 306 (1944)

44. F.H. Mattson, R.A. Volpenhein, JAOCS. 43, 286 (1963)

45. K.W. Miller, D.M. Small, J. Colloid Interfaces. 89, 466 (1982)

46. P. Reis, K. Holmberg, R. Miller, J. Krägel, D. O. Grigoriev, M. E. Leser, H. J. Watzke, Competition between lipase and monoglycerides at interfaces, Langmuir (January 2008), in print

47. W. Tsuzuki, A. Ue, A. Nagao, M. Endo, M. Abe, Biochim. Biophys. Acta 1684, 1 (2004)

48. S. Mun, E.A. Decker, D.J. McClements, Food Res. Int. 40, 770 (2007)

49. M. Carey, O. Hernell, Semin. Gastroint. Dis. 3, 189 (1992)

50. M. Furuhashi, G. Tuncman, C. Görgün, L. Makowski, G. Atsumi, E. Vaillancourt, K. Kono, V. Babaev, S. Fazio, M. Linton, R. Sulsky, J. Robl, R. Parker, G. Hotamisligil, Nature 447, 959 (2007)

51. H. Stam, W.C. Hülsmann, Eur. Heart J. 6, 158 (1985) 